\title{
Safety and Tolerability of Levomilnacipran ER in Major Depressive Disorder: Results from an Open-Label, 48-Week Extension Study
}

\author{
Rajnish Mago • Giovanna Forero • William M. Greenberg • \\ Carl Gommoll $\cdot$ Changzheng Chen
}

Published online: 3 September 2013

(c) The Author(s) 2013. This article is published with open access at Springerlink.com

\begin{abstract}
Background Levomilnacipran (1S, $2 R$-milnacipran) is a potent and selective serotonin (5-HT) and norepinephrine (noradrenaline) reuptake inhibitor approved for the treatment of major depressive disorder in adults.

Objective The objective of this study was to evaluate the longer-term safety and tolerability of levomilnacipran extended-release (ER).

Methods Patients who completed double-blind treatment/ down-taper in one of three lead-in levomilnacipran ER studies were eligible for this 48-week open-label extension. Safety evaluations included assessment of treatmentemergent adverse events (TEAEs), physical examinations, laboratory and vital sign measures, and suicidality, summarized using descriptive statistics for the safety population.

Results The completion rate was $47 \%$; median treatment duration was 280 days. The most frequent reasons for discontinuation were withdrawal of consent $(14 \%)$ and adverse events (AEs; $13 \%$ ). TEAEs were reported by 712 (86\%) patients; most were mild/moderate and occurred early in treatment. The most common TEAEs were headache $(22 \%)$ and nausea (16\%); $36(4 \%)$ patients had $\geq 1$
\end{abstract}

R. Mago $(\bowtie)$

Mood Disorders Program, Department of Psychiatry and Human Behavior, Thomas Jefferson University, 833 Chestnut St., Suite 210 E, Philadelphia, PA 19107, USA

e-mail: rajnish.mago@jefferson.edu

G. Forero · W. M. Greenberg · C. Gommoll Clinical Development, Forest Research Institute, Jersey City, NJ, USA

C. Chen

Biostatistics, Forest Research Institute, Jersey City, NJ, USA serious AEs. No clinically meaningful changes occurred in mean liver enzyme, metabolic, hematologic, urinalysis, or serum values; potentially clinically significant high AST or ALT values ( $\geq 3 \times$ upper limit of normal) occurred in five patients. Vital sign changes occurred early and remained relatively stable. Mean increases for pulse rate $(9.1$ beats per minute $[\mathrm{bpm}])$, and supine systolic $(3.9 \mathrm{mmHg})$ and diastolic $(3.3 \mathrm{mmHg})$ blood pressure were noted. The increase in the mean QT interval corrected using the Bazett formula $(10.9 \mathrm{~ms})$ was consistent with heart rate increase (12.8 bpm); there was no meaningful change in mean QT interval corrected using the Fridericia formula $(-1.3 \mathrm{~ms})$. Other than tachycardia and heart rate increases, ECGrelated TEAEs were low $(<0.5 \%)$.

Conclusion No new or inconsistent safety/tolerability findings were discovered during longer-term evaluation.

\section{Introduction}

Major depressive disorder (MDD) is a commonly recurrent and disabling disorder that can disrupt functioning in multiple domains including work, school, family life, and social relationships. By 2030, unipolar major depression is projected to be among the top three causes of global disease burden [1]. Pharmacologic treatment over an extended period of time is recommended for many patients with MDD to reduce the risk of depression recurrence [2] and associated psychosocial impairment. For pharmacologic treatment to be effective, adherence to antidepressant medication at the recommended dose for the recommended duration is critical. Poor tolerability frequently contributes to issues such as inadequate dose titration and treatment noncompliance, with adverse effects commonly cited as the reason antidepressant medication is discontinued prematurely [3]. As such, it is 
important to assess the safety and tolerability of antidepressant agents over long-term treatment.

Levomilnacipran $(1 S, 2 R$-milnacipran) is a potent and selective serotonin (5-HT) and norepinephrine (noradrenaline) reuptake inhibitor (SNRI) that is approved for the treatment of MDD in adults. An extended-release (ER) formulation was developed to allow for once-daily dosing.

Levomilnacipran has approximately twofold greater potency for inhibition of norepinephrine relative to serotonin reuptake [4]. Potency for inhibition of serotonin reuptake relative to norepinephrine reuptake varies among members of the SNRI class of antidepressants. Duloxetine is tenfold more potent for serotonin reuptake inhibition than norepinephrine reuptake inhibition while venlafaxine and desvenlafaxine show 30-fold higher potency for inhibiting serotonin versus norepinephrine reuptake [5]. For venlafaxine, meaningful norepinephrine inhibition is only achieved at high doses [6], so it primarily acts as a selective serotonin reuptake inhibitor (SSRI) when prescribed at the usual dose of $150 \mathrm{mg} /$ day or less. Compared with duloxetine [4], venlafaxine [4], or desvenlafaxine [7], levomilnacipran has over tenfold higher selectivity for norepinephrine versus serotonin reuptake inhibition.

Since many patients with MDD take multiple medications, the potential for drug-drug interactions is clinically relevant. Levomilnacipran is not extensively hepatically metabolized and is primarily renally excreted unchanged in urine (approximately $58 \%$ of administered dose) [8]. Cytochrome P450 (CYP) 3A4 oxidizes a broad spectrum of drugs, including levomilnacipran ER, by a number of metabolic processes. This may result in pharmacokinetic interactions between drugs due to CYP3A4 inhibition or induction. Dose adjustment is recommended when levomilnacipran ER is co-administered with strong inhibitors of CYP3A4 (e.g., ketoconazole); no dose adjustment is needed when co-administered with a CYP3A4 inducer or substrate $[8,9]$.

Short-term efficacy has been previously demonstrated in four randomized, double-blind studies comparing fixed [10, 11] or flexible doses $[12,13]$ of levomilnacipran ER to placebo. In another flexible-dose study, levomilnacipran ER improved depressive symptoms but did not achieve statistically significant separation from placebo [14]. Additionally, in a relapse prevention study comparing levomilnacipran ER and placebo [15], time to relapse was greater in the levomilnacipran ER group versus placebo, although the treatment difference was not statistically significant. Levomilnacipran ER was generally well-tolerated in all studies.

The primary objective of this open-label extension study was to evaluate the longer-term safety and tolerability of levomilnacipran ER in the treatment of adult patients with MDD. Although efficacy assessments were collected, they were not categorized as primary, secondary, or additional outcomes and inferential statistics were not performed.

\section{Methods}

This Phase III clinical study (NCT01034267) was conducted between December 2009 and June 2012 at 68 study centers in the US. The study was in full compliance with US FDA guidelines for Good Clinical Practice and the ethical principles of the Declaration of Helsinki. The final protocol was approved by the appropriate institutional review board (IRB) for each study site and patients gave written informed consent for their participation.

\subsection{Study Design}

This study was a multicenter, open-label, flexible-dose (40-120 mg/day) extension study of levomilnacipran ER conducted in adult patients with MDD. Participants were required to have completed 8 weeks of fixed- or flexibledose treatment with levomilnacipran ER or placebo, and the 2-week double-blind down-taper period in one of three double-blind lead-in studies. The lead-in studies were study 1 (NCT00969709; levomilnacipran ER 40, 80, or $120 \mathrm{mg} /$ day or placebo) [10]; study 2 (NCT00969150; levomilnacipran ER 40-120 mg/day or placebo) [14]; and study 3 (NCT01034462; levomilnacipran ER 40-120 mg/ day or placebo) [13].

This extension study was 52 weeks in duration and consisted of a 48 -week open-label treatment period followed by a down-taper period of up to 4 weeks (Fig. 1); week 0/visit 1 refers to the last visit of the double-blind down-taper, which was also the first week of the open-label study. There were 18 scheduled clinic visits during the open-label treatment (weeks 0-48) and down-taper (weeks 48-52) periods; visits occurred weekly for the first 4 weeks and every 4 weeks thereafter. Additionally, 11 interim telephone contacts were conducted at 2-week intervals between site visits to evaluate adverse events (AEs) and concomitant medications.

All patients received levomilnacipran ER $20 \mathrm{mg}$ on days 1 and 2, and $40 \mathrm{mg}$ on days 3-7 of open-label treatment. After week 1, the dose could be increased in 40-mg increments at weekly intervals based on the investigator's judgment of response and the absence of dose-limiting AEs. The minimum and maximum allowable dosages were 40 and $120 \mathrm{mg} /$ day, respectively. Following a dose increase, the dose could be decreased to the previous level at any time if, in the opinion of the investigator, doselimiting AEs developed. Patients completing 48 weeks of open-label treatment or prematurely discontinuing underwent a dosage down-taper period of up to 4 weeks if 


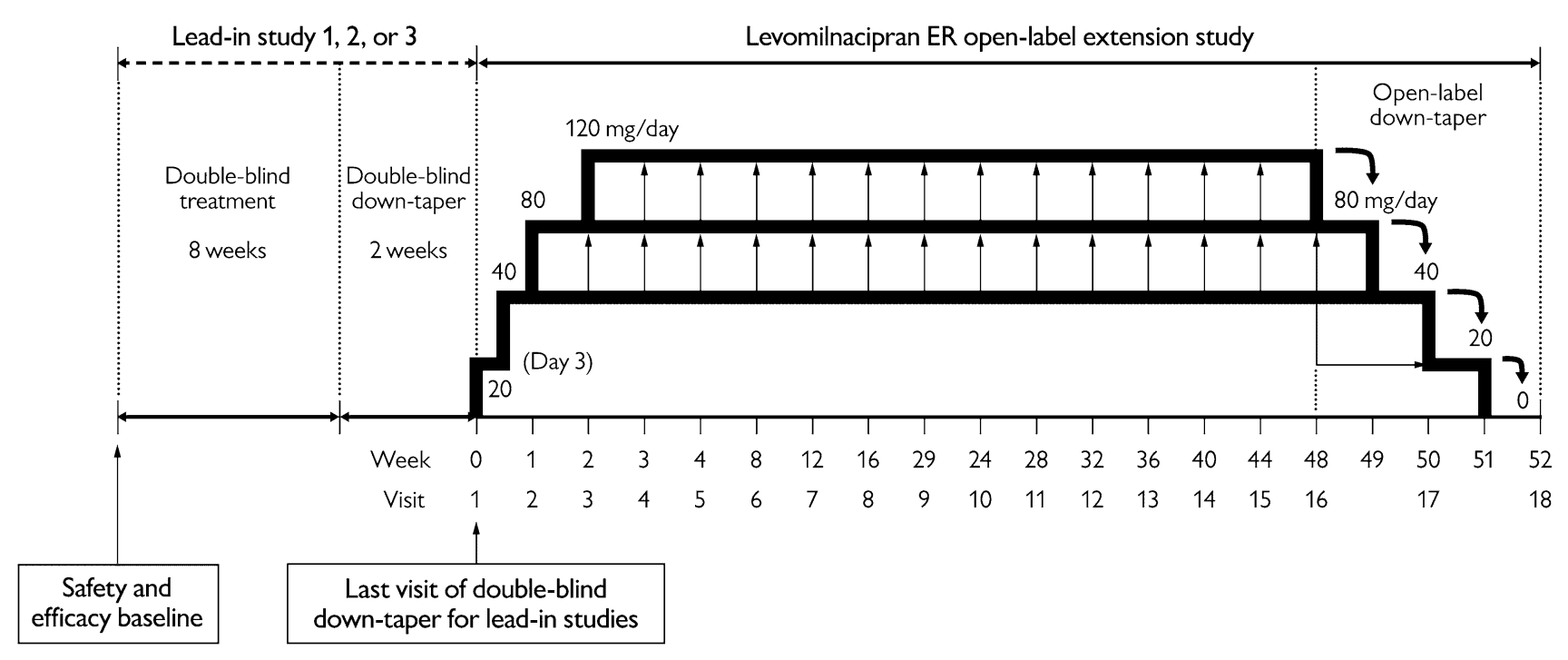

Fig. 1 Study design. $E R$ extended-release

medically appropriate. Final dose levels were tapered by weekly decrements as follows: final $120 \mathrm{mg} / \mathrm{day}$ to $80 \mathrm{mg} / \mathrm{day}, 40 \mathrm{mg} / \mathrm{day}, 20 \mathrm{mg} /$ day, and 0 ; final $80 \mathrm{mg} / \mathrm{day}$ to $40 \mathrm{mg} / \mathrm{day}, 40 \mathrm{mg} / \mathrm{day}, 20 \mathrm{mg} / \mathrm{day}$ and 0 ; final $40 \mathrm{mg}$ / day to $20 \mathrm{mg} /$ day, $20 \mathrm{mg} /$ day, 0 and 0 .

\subsection{Inclusion Criteria}

Inclusion criteria for the lead-in studies $[10,13,14]$ were similar and typical of criteria used in clinical studies of antidepressants for the treatment of MDD. At the lead-in study screening visit, all patients were between 18 and 80 years of age, inclusive, and met the criteria for MDD defined by the Diagnostic and Statistical Manual of Mental Disorders, 4th edition, text revision, (DSM-IV-TR) [16]. Included patients had an ongoing depressive episode of $\geq 4$ weeks' (study 2 and 3 ) or $\geq 8$ weeks' (study 1 ) duration and a score $\geq 30$ on the clinician-rated MontgomeryÅsberg Depression Rating Scale (MADRS) [17].

To be included in the extension study, patients had to have completed double-blind treatment with levomilnacipran ER or placebo and the double-blind down-taper period in one of the lead-in studies. At the screening visit of the lead-in study, patients had normal physical examination findings, clinical laboratory test results, and ECG results, or abnormal results that were judged not clinically significant by the investigator. Additionally, women of childbearing potential had a negative serum $\beta$-human chorionic gonadotropin ( $\beta$-hCG) serum pregnancy test and a negative urine pregnancy test at week 0 of open-label treatment.

\subsection{Exclusion Criteria}

Psychiatric or medical exclusions during the lead-in studies were similar and typical of exclusions in antidepressant clinical trials for MDD. Psychiatric exclusions included a history of meeting DSM-IV-TR criteria for a manic/hypomanic episode or depressive episode with psychotic features, or substance abuse or dependence within the preceding 6 months (except nicotine or caffeine). Medical exclusions included concurrent medical conditions that might interfere with the conduct of the study, confound the interpretation of study results, or endanger the patient's well-being. Patients were excluded if they met any of the following criteria for suicide risk: investigator's determination of significant risk; a score $\geq 5$ on MADRS Item 10 (Suicidal Thoughts) at the final down-taper period visit of the lead-in study; or significant risk based on information collected from patient interviews and the Columbia-Suicide Severity Rating Scale (C-SSRS) [18]. Patients requiring prohibited concomitant medications were excluded; any concomitant drug with psychotropic activity, except eszopiclone, zolpidem, zolpidem ER, or zaleplon for sleep, was prohibited.

Any exclusionary psychiatric or medical condition that developed during the lead-in study resulted in exclusion from the extension study. At week 0 of the open-label study, patients with any clinically significant, unstable, or decompensated cardiovascular change or a positive urine drug screen for alcohol or any other illegal or prohibited substances were excluded. 


\subsection{Safety and Tolerability Measures}

Safety assessments were performed at regular intervals. AEs were spontaneously reported by the patient or identified by study personnel and coded by the preferred Medical Dictionary for Regulatory Activities (MedDRA) term (weeks 0-48 and during down-taper/until 30 days after the last dose of treatment). AEs were evaluated by the investigator for intensity (i.e., mild, moderate, or severe) and possible relationship to treatment (related or not related). Other evaluations included physical examinations (week 24 and 48), clinical laboratory values (weeks $0,16,24,36$, 48), ECGs (weeks 0, 2, 4, 8, 16, 24, 36, 48), and vital sign assessment (all scheduled visits in weeks 1-48 and downtaper weeks 50 and 52).

Severity of suicidal ideation and behavior were reported from C-SSRS evaluations (weeks 1-48 and down-taper weeks 50 and 52); suicide-related AEs were also examined. On the C-SSRS, 5-item scales classify the severity of suicidal ideation from 1 (wish to be dead, without intent to act) to 5 (active suicidal ideation with specific plan and intent), and suicidal behavior from 0 (no suicidal behavior) to 4 (actual attempt).

Ongoing AEs and concomitant medications being taken at the final visit of the double-blind down-taper period of the lead-in study were transferred to the case report form for week 0 of the open-label study.

\subsection{Efficacy Measures}

The primary objective of this study was the evaluation of long-term safety and tolerability of levomilnacipran ER; as such, efficacy assessments were collected but not categorized as primary, secondary, or additional outcomes. Collected measures consisted of the MADRS (weeks 0, 2-8, 16, 24, 36, 48), Clinical Global Impressions-Severity (CGI-S) (weeks 0-48), and Clinical Global ImpressionsImprovement (CGI-I) [19] (weeks 1-48); the CGI-I was rated with respect to the baseline assessment of the lead-in study.

\subsection{Data Analysis}

The enrolled population consisted of all patients who completed the double-blind and down-taper periods of a lead-in study and consented to participate in the open-label extension study of levomilnacipran ER. Safety analyses were based on the safety population, which consisted of enrolled patients who had at least one dose of open-label levomilnacipran ER during the extension study. Patient disposition was presented by number and percentage for the safety population; extent of exposure, daily dose, and safety parameters were summarized using descriptive statistics. Potentially clinically significant (PCS) high and low criteria for laboratory values, ECG, and vital signs were prespecified. Baseline values from the respective lead-in study were used as the baseline for all analyses of safety parameters.

Baseline for all efficacy analyses was the corresponding baseline from the respective lead-in study; change in MADRS total score was also summarized using week 0 of the extension study as baseline. Efficacy summaries included MADRS total score change from baseline (lead-in study) and week 0 (open-label extension), CGI-S score change from baseline, CGI-I score, MADRS response (MADRS score $\geq 50 \%$ reduction from baseline) and remission (MADRS score $\leq 10$ ) rates, and CGI-I response rate (CGI-I score $\leq 2$ ). Evaluations were based on the modified intent-to-treat (ITT) population, which consisted of all patients in the safety population who had at least one MADRS total score assessment during the extension study. No inferential statistical analyses were performed; descriptive statistics were presented by visit for all efficacy parameters using the last observation carried forward (LOCF) approach to impute missing values after week 1, and the observed cases (OC) approach.

\section{Results}

\subsection{Patient Disposition and Demographic Characteristics}

A total of 828 patients were enrolled in the open-label extension (enrolled population); 825 patients received at least one dose of open-label levomilnacipran ER (safety population) and 813 patients additionally had at least one post-baseline MADRS assessment during open-label treatment (ITT population). For patients who entered the extension study and were included in the safety population, 469 had received levomilnacipran ER in a respective leadin study and 356 had received placebo (Table 1).

All patients completed a 2-week double-blind downtaper before entering the extension study; each patient received levomilnacipran ER on an identical progressive dose-titration schedule in the extension study. Patients who completed 48 weeks of open-label treatment were considered extension study completers; reasons for discontinuation are presented in Table 2. AEs that led to the discontinuation of three or more patients were nausea (ten patients); hyperhidrosis (eight patients); tachycardia (seven patients); hypertension (six patients); headache (five patients); suicide attempt (two patients with actual attempts and two patients with aborted attempts); urinary hesitation and depression (four patients each); and urinary retention and blood pressure increase (three patients each). 
Table 1 Lead-in study treatment assignment and final dose for patients who entered the extension study

\begin{tabular}{lllll}
\hline Lead-in study & Placebo & \multicolumn{2}{l}{ Levomilnacipran ER } & $80 \mathrm{mg} / \mathrm{day}$ \\
\cline { 3 - 5 } & & $40 \mathrm{mg} / \mathrm{day}$ & 83 & $120 \mathrm{mg} / \mathrm{day}$ \\
\hline Study 1 (assigned fixed-dose) & 107 & 97 & 23 & 89 \\
Study 2 (final flexible dose) & 125 & 29 & 26 & 46 \\
Study 3 (final flexible dose) & 124 & 26 & 48 \\
\hline
\end{tabular}

$E R$ extended-release

${ }^{\text {a }}$ In study 3, a final daily levomilnacipran ER dose of 20 or $160 \mathrm{mg}$ was inadvertently taken by one patient each; these two patients were not included in the final dose count

Table 2 Premature discontinuation during extension study (safety population) of levomilnacipran ER $40-120 \mathrm{mg} /$ day $(n=825)$

\begin{tabular}{lc}
\hline Patient status & $n(\%)$ \\
\hline Completed open-label treatment & $384(46.5)$ \\
Prematurely discontinued & $441(53.5)$ \\
Reason for discontinuation & \\
Adverse event & $107(13.0)$ \\
Insufficient therapeutic response & $56(6.8)$ \\
Protocol violation & $67(8.1)$ \\
Withdrawal of consent & $118(14.3)$ \\
Lost to follow-up & $87(10.5)$ \\
Other reasons & $6(0.7)$ \\
Entered down-taper period & \\
\end{tabular}

ER extended-release

${ }^{\text {a }}$ Patients completing 48 weeks of open-label treatment or prematurely discontinuing entered a dosage down-taper period of up to 4 weeks if medically appropriate

Mean (standard deviation [SD]) patient age was 44 (12.8) years and $65 \%$ of the patients were women; mean body mass index was $29.10(5.57) \mathrm{kg} / \mathrm{m}^{2}$ (safety population). The majority of patients had a history of recurrent MDD (78 \%), with a 12-year mean duration of illness; the mean (SD) duration of the current episode was 20 (51) months (median duration 8 months).

At baseline, 54 and $22 \%$ of patients had a lifetime history of suicidal ideation and suicidal behavior, respectively, as determined by the C-SSRS. No patients were considered to represent a significant suicide risk at the time of study enrollment and no patient had made a suicide attempt within the past year.

\subsection{Extent of Exposure}

The median duration of treatment was 280 days. The final daily dose was $40 \mathrm{mg} /$ day for $27 \%, 80 \mathrm{mg} /$ day for $26 \%$, and $120 \mathrm{mg} /$ day for $47 \%$ of patients. For $0.4 \%$ of patients, the final daily dose was $20 \mathrm{mg} /$ day; these patients discontinued the study during the up-titration period but were included in the safety analysis. Patient-years of exposure was 502.3.

\subsection{Adverse Events}

During open-label treatment, $712(86 \%)$ patients reported a treatment-emergent AE (TEAE). The number of patients who discontinued due to AEs in this extension study was similar for patients who had received double-blind lead-in placebo (55 patients [11\%]) or levomilnacipran ER (52 patients [15\%]) treatment. The incidence of patients with at least one TEAE report decreased over time during openlabel treatment (Fig. 2).

The most common TEAEs ( $\geq 5 \%$ of patients during open-label treatment) are presented in Table 3. Most TEAEs were considered mild (57\%) or moderate $(38 \%)$; of the total number of TEAEs reported $(n=3,408), 49 \%$ were considered to be possibly or probably related to levomilnacipran ER. Of TEAEs that were considered severe $(5 \%)$, the most common were nausea and headache. Most TEAEs were transient in nature. Of patients with at least one TEAE that was considered possibly or probably related to levomilnacipran ER $(n=557), 35 \%$ had events that were considered mild and $55 \%$ had events that were considered moderate in severity.

Pregnancy was reported in three patients during openlabel treatment and resulted in premature discontinuation

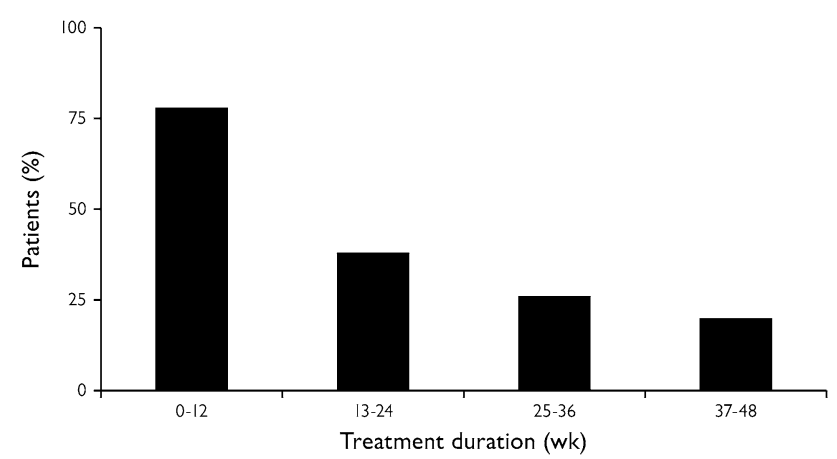

Fig. 2 Incidence of patients with $\geq 1$ treatment-emergent adverse event over time during open-label treatment (safety population) 
Table 3 Most common treatment-emergent adverse events ( $\geq 5 \%$ of patients) during open-label treatment (safety population) of levomilnacipran ER 40-120 mg/day $(n=825)$

\begin{tabular}{|c|c|c|}
\hline TEAEs & $n(\%)$ & $\begin{array}{l}\text { Related to } \\
\text { levomilnacipran } \\
\mathrm{ER}^{\mathrm{a}}[n(\%)]\end{array}$ \\
\hline Patients with $\geq 1$ TEAE & $712(86.3)$ & $557(67.5)$ \\
\hline Headache & $183(22.2)$ & $116(14.1)$ \\
\hline Nausea & $134(16.2)$ & $100(12.1)$ \\
\hline Upper respiratory tract infection & 109 (13.2) & $1(0.1)$ \\
\hline Hyperhidrosis & $90(10.9)$ & $86(10.4)$ \\
\hline Constipation & $79(9.6)$ & $61(7.4)$ \\
\hline Nasopharyngitis & $70(8.5)$ & $2(0.2)$ \\
\hline Dizziness & $67(8.1)$ & $54(6.5)$ \\
\hline Insomnia & $66(8.0)$ & $47(5.7)$ \\
\hline Tachycardia & $65(7.9)$ & $63(7.6)$ \\
\hline Dry mouth & $59(7.2)$ & $54(6.5)$ \\
\hline Heart rate increased & $55(6.7)$ & $53(6.4)$ \\
\hline Hypertension & $53(6.4)$ & $35(4.2)$ \\
\hline Back pain & $46(5.6)$ & $3(0.4)$ \\
\hline Erectile dysfunction $^{\mathrm{b}}$ & $16(5.6)$ & $15(5.2)$ \\
\hline Blood pressure increased & $44(5.3)$ & $37(4.5)$ \\
\hline
\end{tabular}

$E R$ extended-release, TEAE treatment-emergent adverse event

${ }^{\text {a }}$ Percentage of safety population with TEAE possibly or probably related to levomilnacipran ER as determined by investigator assessment

b Percentage relative to the number of male patients $(n=286)$

of two of the patients. The patient who did not discontinue the study prematurely had received treatment for 330 days and had a positive pregnancy test on day 337. All three pregnancies resulted in live births with no complications.

During open-label treatment, 36 (4\%) patients had at least one serious AE (SAE). No deaths occurred during this study; however, one patient who completed 8 weeks of placebo treatment in a lead-in study and received openlabel levomilnacipran ER for 224 days was diagnosed with stage IV gastric adenocarcinoma on day 223. This patient discontinued from the study and died from the cancer 42 days later; this SAE was not considered by the investigator to be related to levomilnacipran ER.

SAEs resulted in the discontinuation of $13(2 \%)$ patients. SAEs that were considered possibly or probably related to levomilnacipran ER and resulted in premature discontinuation from the study occurred in four patients (angina pectoris/heart rate increased, supraventricular extrasystoles/tachycardia/ventricular extrasystoles, convulsion/encephalopathy, and mania in one patient each). SAEs that were considered not related to levomilnacipran ER and resulted in premature discontinuation occurred in nine patients (depression, chest pain [unknown etiology], overdose/suicide attempt, gastric cancer stage IV, pharyngeal cellulitis, depression/suicidal ideation, lower limb fracture, suicidal behavior, and overdose/suicide attempt in one patient each).

During the down-taper period, $75(9 \%)$ patients reported newly emergent AEs. SAEs occurred in three $(0.4 \%)$ patients during this period (colitis, obstructive inguinal hernia, and spinal operation in one patient each). No SAE during the down-taper period was considered possibly or probably related to levomilnacipran ER.

\subsection{Baseline to Endpoint: Safety Parameter Changes Over Time}

\subsubsection{ALT, AST, and Liver Function Tests}

No clinically meaningful mean changes or consistent trends were noted in any liver enzyme value. The mean (SD) change from baseline to endpoint was 2.2 (13.4) U/L for ALT, 2.1 (11.0) U/L for AST, 2.3 (12.9) U/L for alkaline phosphatase, and $-1.0(3.8) \mu \mathrm{mol} / \mathrm{L}$ for bilirubin. No patient met Hy's Law criteria (ALT or AST $\geq 3 \times$ upper limit of normal [ULN], total bilirubin $>2 \times$ ULN, and alkaline phosphatase $<2 \times$ ULN) [20].

PCS high AST/ALT values $(\geq 3 \times \mathrm{UNL})$ were noted in five patients (one ALT, four AST; organ source of enzymes not determined). No patient met PCS criteria for low albumin $(<0.9 \times \mathrm{ULN})$, total bilirubin $(>1.5 \times \mathrm{ULN})$, or alkaline phosphatase $(\geq 3 \times \mathrm{ULN})$. On day 115 , one patient had an AST value of $330 \mathrm{U} / \mathrm{L}(\geq 5 \times \mathrm{ULN})$ and elevated ALT (129 U/L, not PCS); both AST and ALT normalized while the patient continued open-label treatment and no associated TEAEs were reported. PCS high liver enzyme laboratory values were reported as TEAEs in two patients (ALT/AST increased and liver function test abnormal in one patient each). Neither of these TEAEs was an SAE or led to study discontinuation and values decreased to normal levels while patients remained on levomilnacipran ER in open-label or down-taper treatment.

Elevated, but non-PCS, changes in ALT or AST values also resulted in the discontinuation of two patients who had received placebo in the respective lead-in studies. In one patient, TEAEs of increased AST and ALT occurred during double-blind down taper in the lead-in study; the patient subsequently received 124 days of open-label treatment and was discontinued from the study due to ongoing increased AST and ALT values that were considered possibly or probably related to levomilnacipran ER. Additionally, one patient who received open-label levomilnacipran ER for 3 days developed a TEAE of increased AST on day 1 and was discontinued from the 
study; the AE resolved on day 10 and was considered related to levomilnacipran ER.

\subsubsection{Metabolic Parameters and Clinical Laboratory Evaluations}

No clinically meaningful trends or changes were noted for mean values of metabolic, hematologic, urinalysis, or other serum parameters from baseline to endpoint; relatively small proportions of patients shifted from normal baseline values to low, high, or PCS values for these parameters.

\subsubsection{Vital Signs}

In long-term, open-label treatment, vital sign changes occurred early and values then remained relatively stable over the course of the study. Increase (mean [SD]) from baseline to endpoint was noted for pulse rate (9.1 [11.6] beats per minute [bpm]), and supine systolic (3.9 [11.6] $\mathrm{mmHg}$ ) and diastolic (3.3 [8.8] $\mathrm{mmHg}$ ) blood pressure; median increases for pulse rate, systolic blood pressure, and diastolic blood pressure were $8.0 \mathrm{bpm}$, $4.0 \mathrm{mmHg}$, and $2.5 \mathrm{mmHg}$, respectively. For change in pulse rate and blood pressure, differences in age $(<45, \geq 45$ to $<60, \geq 60$ years), race (black/African American and other), and sex (male and female) subgroups compared with the total population were small and not clinically meaningful. In general, PCS vital signs were noted in $<1 \%$ of patients (Table 4). No patients met PCS criterion for high systolic blood pressure; PCS criterion for high diastolic blood pressure was met in $2 \%$ of patients.

Changes in pulse rate, supine systolic blood pressure, and supine diastolic blood pressure analyzed by final daily dose are shown in Table 5. Changes in these vital signs by final daily dose should be interpreted with caution since levomilnacipran ER dose could be adjusted at any time during the extension study; as such, the final dose may not be representative of the dose taken during the entire extension or for the longest period of time.

Orthostatic hypotension (reduction $\geq 20 \mathrm{mmHg}$ in systolic blood pressure or a reduction of $\geq 10 \mathrm{mmHg}$ in diastolic blood pressure while changing from supine to standing position) occurred in $21.1 \%$ of patients on at least one occasion during the open-label treatment period. Overall, levomilnacipran ER was weight neutral throughout the study, with mean (SD) change in body weight of -0.55 (4.34) $\mathrm{kg}$ from baseline to endpoint (Fig. 3). Weight change meeting PCS criteria of $\geq 7 \%$ increase or $\geq 7 \%$ decrease occurred in 10 and $17 \%$ of patients, respectively. Vital sign changes from baseline to endpoint were consistent with results from acute studies of levomilnacipran ER.

\subsubsection{Electrocardiography}

An increase (mean [SD]) in QT interval corrected for heart rate using the Bazett formula (QTcB) from baseline to endpoint of open-label treatment (10.9 [20.9] ms) was observed; this was consistent with the increase noted in heart rate $(12.8$ [12.3] bpm). No meaningful change in mean QT interval corrected for heart rate using the Fridericia formula $(\mathrm{QTcF})(-1.3$ [17.4] ms) was observed.

The percentage of patients with ECG-related TEAEs other than tachycardia and increased heart rate were low $(<0.5 \%)$ and resulted in study discontinuation for three patients (supraventricular extrasystoles/ventricular extrasystoles, ECG T-wave inversion, and arrhythmia in one patient each). SAEs related to ECG abnormalities were reported in two patients (supraventricular extrasystoles/ ventricular extrasystoles and ventricular extrasystoles in one patient each). ECG values that met PCS criteria were noted in three patients: PR interval $(\geq 250 \mathrm{~ms})$, QRS interval $(\geq 150 \mathrm{~ms})$, and QTcB $(>500 \mathrm{~ms})$ in one patient each. No patient had a PCS value for QTcF $(>500 \mathrm{~ms})$ and no QTcF-related TEAEs were reported.

\subsection{Suicidality}

\subsubsection{Columbia-Suicide Severity Rating Scale}

During open-label treatment, $22 \%$ of patients reported C-SSRS-based suicidal ideation; the least severe category of suicidal ideation (wish to be dead, without intent to act) was the most common ideation category reported (13\%). Suicidal behavior was reported for three $(0.4 \%)$ patients (one actual attempt and two aborted attempts).

\subsubsection{Suicide-Related Adverse Events}

A total of eight (1\%) patients had TEAEs associated with suicidality: suicidal ideation in four patients and suicidal behavior in four patients (aborted attempt in two patients and actual attempt in two patients). These suicide-related TEAEs were reported as an SAE and/or contributed to premature discontinuation from the study for seven of the eight patients.

\subsection{Efficacy Summary}

Efficacy assessments were collected but no inferential statistics were performed. Improvements observed with double-blind levomilnacipran ER in the lead-in studies were sustained during open-label treatment. Decrease in MADRS score was seen from the baseline of the respective lead-in study to the beginning of the extension study (week 0 ), from week 0 to week 48 of the extension trial, and from 
Table 4 Potentially clinically significant vital sign values during the open-label treatment period (safety population) of levomilnacipran ER $40-120 \mathrm{mg} /$ day $(\mathrm{n}=822)^{\mathrm{a}}$

\begin{tabular}{lll}
\hline Vital sign, unit & PCS criteria & $n(\%)$ \\
\hline Supine systolic blood pressure, $\mathrm{mmHg}$ & High $(\geq 180 \mathrm{mmHg}$ and increase $\geq 20 \mathrm{mmHg})$ & 0 \\
& Low $(\leq 90 \mathrm{mmHg}$ and decrease $\geq 20 \mathrm{mmHg})$ & $3(0.4)$ \\
Supine diastolic blood pressure, $\mathrm{mmHg}$ & High $(\geq 105 \mathrm{mmHg}$ and increase $\geq 15 \mathrm{mmHg})$ & $19(2.3)$ \\
& Low $(\leq 50 \mathrm{mmHg}$ and decrease $\geq 15 \mathrm{mmHg})$ & $1(0.1)$ \\
Supine pulse rate, bpm & High $(\geq 120 \mathrm{bpm}$ and increase $\geq 15 \mathrm{bpm})$ & $4(0.5)$ \\
& Low $(\leq 50 \mathrm{bpm}$ and decrease $\geq 15 \mathrm{bpm})$ & $2(0.2)$ \\
\hline
\end{tabular}

bpm beats per minute, $E R$ extended-release, $P C S$ potentially clinically significant

${ }^{a}$ Number of patients in safety population $(n=825)$ with a baseline and at least one postbaseline value

Table 5 Vital sign changes by final daily levomilnacipran extended-release dose

\begin{tabular}{llll}
\hline Vital sign parameter & \multicolumn{2}{l}{ Levomilnacipran ER final daily dose ${ }^{\mathrm{a}}$} & \\
\cline { 2 - 4 } & $40 \mathrm{mg} /$ day $(n=223)$ & $80 \mathrm{mg} /$ day $(n=212)$ & $120 \mathrm{mg} / \mathrm{day}(n=387)$ \\
\hline Pulse rate, bpm & $8.0(11.2)$ & $8.3(12.0)$ & $10.1(11.4)$ \\
Mean (SD) & $8.0(-28,47)$ & $7.5(-20,46)$ & $10.0(-17,46)$ \\
Median (min, max) & $3.2(11.7)$ & $4.2(11.4)$ & $4.3(11.7)$ \\
Supine systolic blood pressure, $\mathrm{mmHg}$ & $4.0(-26,40)$ & $4.0(-25,42)$ \\
Mean (SD) & $2.0(-30,36)$ & & $3.2(9.0)$ \\
Median (min, max) & $3.2(8.5)$ & $3.7(8.9)$ & $2.0(-17,39)$ \\
Supine diastolic blood pressure, $\mathrm{mmHg}$ & $4.0(-18,24)$ &
\end{tabular}

bpm beats per minute, $E R$ extended-release, max maximum, min minimum, $S D$ standard deviation

${ }^{a}$ Levomilnacipran ER dose could be adjusted at any time during the extension study so the final dose may not be representative of the dose taken during the entire extension or for the longest period of time

Fig. 3 Mean change in body weight by week (safety population). Week 0 is also the final visit of the double-blind down-taper period of the lead-in study

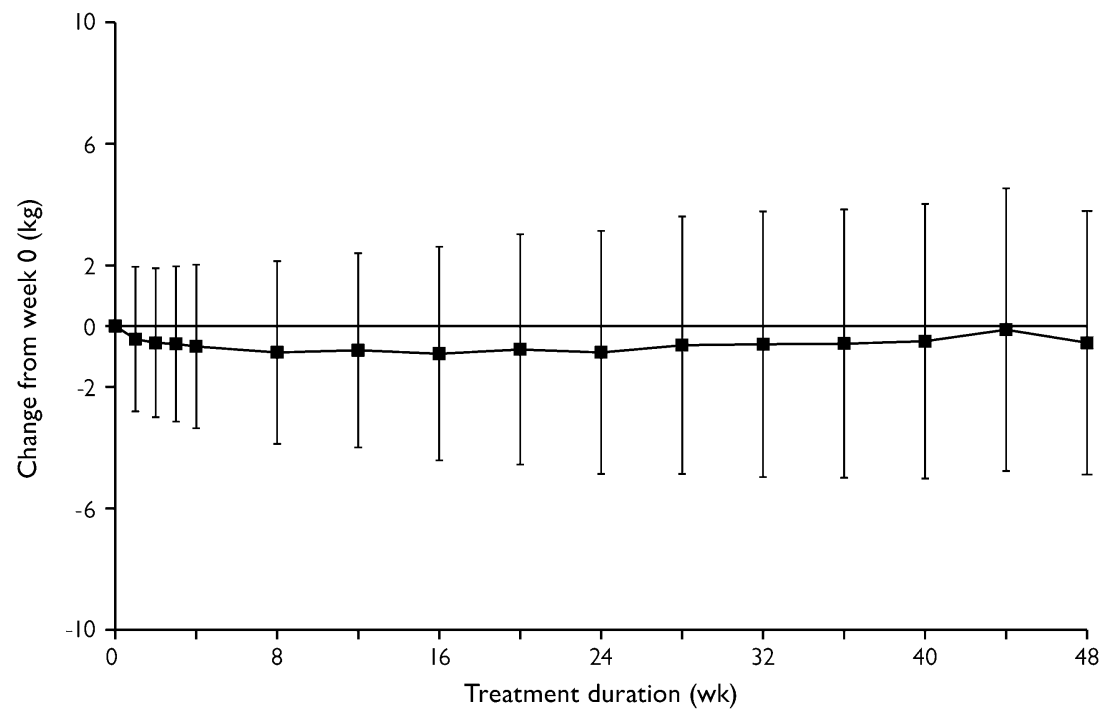


Table 6 Efficacy assessments (intent-to-treat population) of levomilnacipran ER 40-120 $\mathrm{mg} /$ day $(n=813)$

CGI-I Clinical Global Impressions-Improvement, CGI-S Clinical Global

Impressions-Severity, $E R$ extended-release, $L O C F$ last observation carried forward, MADRS Montgomery-Åsberg Depression Rating Scale, $O C$ observed cases, $S D$ standard deviation

${ }^{\text {a }}$ Week 0 of the open-label study corresponds to the final visit in the double-blind lead-in study

${ }^{b}$ CGI-I rating was based on baseline of the respective double-blind lead-in study

\begin{tabular}{llr}
\hline Efficacy measure & & Mean (SD) \\
\hline MADRS total score & Baseline of double-blind lead-in study & $35.9(4.1)$ \\
LOCF & Change from baseline (lead-in) to open-label week $0^{\mathrm{a}}$ & $-13.5(10.7)$ \\
& Change from week 0 $0^{\mathrm{a}}$ to week 48 & $-10.0(11.6)$ \\
& Change from baseline (lead-in) to week 48 & $-23.6(10.8)$ \\
OC & Change from baseline (lead-in) to open-label week $0^{\mathrm{a}}$ & $-13.5(10.7)$ \\
& Change from week 0 $0^{\mathrm{a}}$ to week 48 & $-13.3(10.9)$ \\
& Change from baseline (lead-in) to week 48 & $-27.6(8.5)$ \\
CGI-S & Baseline of double-blind lead-in study & $4.8(0.6)$ \\
LOCF & Change from baseline (lead-in) to open-label week $0^{\mathrm{a}}$ & $-1.3(1.3)$ \\
& Change from baseline (lead-in) to week 48 & $-2.5(1.4)$ \\
OC & Change from baseline (lead-in) to open-label week $0^{\mathrm{a}}$ & $-1.3(1.3)$ \\
& Change from baseline (lead-in) to week 48 & $-3.0(1.1)$ \\
CGI-I & & \\
LOCF & Score at week 48 & $1.9(1.1)$ \\
OC & Score at week 48 & $1.5(0.8)$ \\
\hline
\end{tabular}

baseline of the lead-in study to week 48 (Table 6). Decreased scores from baseline were also seen on other measures of efficacy (Table 6).

The percentage of patients that met criteria for MADRS response $(50 \%$ reduction in lead-in study MADRS baseline total score) at week 48 was high (LOCF $73 \%$, OC $88 \%)$; similar response rates were seen using the CGI-I (CGI-I score $\leq 2)$ (LOCF $75 \%$, OC $90 \%)$. More than half of patients achieved remission (MADRS total score $\leq 10$ ) at week 48 (LOCF $53 \%$, OC $69 \%$ ). Rates of response and remission remained stable from week 24 to week 48 of open-label treatment.

\section{Discussion}

This open-label extension study was conducted to evaluate the longer-term safety and tolerability of levomilnacipran ER in the treatment of adult patients with MDD. No new or inconsistent findings were discovered during this long-term safety evaluation; safety and tolerability outcomes were consistent with results of short-term treatment studies of levomilnacipran ER.

The completion rate in this 48-week extension study was $47 \% ; 13 \%$ of patients discontinued the study due to AEs, which supports the tolerability of levomilnacipran ER for longer-term use in the treatment of a major depressive episode. Although approximately $86 \%$ of patients reported TEAEs during open-label treatment, the incidence of patients with $\geq 1$ TEAE report decreased over time during open-label treatment and most events were considered mild or moderate in intensity. Common TEAEs that were considered to be related to levomilnacipran ER were consistent with its mechanisms of action. In a study of this duration it may be expected that depressive symptoms may worsen in some patients over time; $7 \%$ of patients prematurely discontinued this study due to insufficient therapeutic response.

Since differences in efficacy and tolerability have been demonstrated among the second-generation antidepressant medications (e.g., SSRIs, SNRIs) [21], short- and longterm treatment decisions should be made with safety, tolerability, and effectiveness in mind. In this open-label extension study, longer-term tolerability of levomilnacipran ER was similar to what has been previously observed in acute studies of levomilnacipran ER [10-14]. The most common TEAEs, headache (22\%) and nausea (16\%), were reported more frequently in the extension study by patients who had been assigned to placebo in the respective lead-in study. Nausea was reported in $20 \%$ of patients who received lead-in placebo and $13 \%$ of patients who received lead-in levomilnacipran ER; headache was reported by 25 and $20 \%$ of lead-in placebo- and levomilnacipran ER-treated patients, respectively. The higher incidence of these TEAEs in lead-in placebo patients may reflect the lack of prior exposure to levomilnacipran ER for placebo patients.

Certain AEs, such as sexual dysfunction and weight gain, are more frequently associated with poor treatment adherence and premature discontinuation from antidepressant medication [3]. Although the lack of a formal sexual dysfunction measure in this study prevents a complete evaluation of possible sexual AEs, erectile dysfunction was reported as a TEAE in $6 \%$ of males and there were no discontinuations from the study due to sexual dysfunction. The overall weight-neutral profile of levomilnacipran ER observed in the short-term studies [10-14] was also seen with longer-term use in the extension study. A small mean 
weight loss from baseline to endpoint $(-0.55 \mathrm{~kg})$ was observed and only one patient $(0.1 \%)$ discontinued the study due to weight gain. PCS changes in transaminases and liver function tests, metabolic parameters, vital signs, and ECG were generally small and not clinically meaningful; no patient met the criteria for Hy's Law, a reassuring finding in a long-term study.

As noted in the baseline demographic characteristics of the study population, $54 \%$ of patients had a lifetime history of suicidal ideation and $22 \%$ had a history of suicidal behavior as determined by the C-SSRS. During the extension study, suicidality was assessed by both the C-SSRS and investigator-determined suicide-related TEAEs, which are different types of measures that may yield somewhat discrepant results. The C-SSRS was completed by the patient via an interactive voice response system at every visit; responses were evaluated at that visit for suicidal ideation and behavior that occurred since the last visit. Information collected from the C-SSRS, clinical progress notes, and other rating scales administered during the study (e.g., MADRS) were also reviewed and evaluated by the investigator as part of the overall assessment of suicidality; the totality of this information was used to determine if an event needed to be recorded as a TEAE. In the extension study, the incidence of TEAEs related to suicidal ideation, behavior, or attempt was low; TEAEs of aborted suicide attempt and actual attempt were reported in two patients each. C-SSRS-based suicidal ideation was reported in $22 \%$ of patients, most in the least severe category, and suicidal behavior was reported in three patients (one attempt and two aborted attempts). Each suicide event should be interpreted within the context of the method used to report it.

Although the primary objective of this open-label study was to evaluate the long-term safety and tolerability of levomilnacipran ER, efficacy assessments were collected and summaries were provided; no inferential statistics were performed. Without a placebo or active-control group for comparison, no conclusion regarding the efficacy of levomilnacipran ER in long-term use can be made. Nevertheless, it should be noted that MADRS and CGI-S scores decreased over the course of the study. For patients who completed the study, approximately $90 \%$ responded to treatment and almost $70 \%$ achieved remission. Some discontinuity in treatment effect may have occurred during the double-blind down-titration period since all patients were removed from treatment at this time.

\section{Conclusion}

A good safety and tolerability profile is especially important for antidepressant agents since extended treatment may be prescribed and patients often discontinue antidepressant medications earlier than recommended. Although the lack of placebo control limits the ability to assess the cause of change in an open-label study, the findings in this 48 -week extension study of levomilnacipran ER supported the safety and tolerability findings that were observed in patients who completed a short-term double-blind lead-in study.

Acknowledgments This study was supported by funding from Forest Laboratories, Inc. (New York, NY, USA). Forest Laboratories, Inc. was involved in the study design, collection (via contracted clinical investigator sites), analysis and interpretation of data, and the decision to present these results. Dr. Mago acknowledges the following potential conflict of interest in the past: receiving consulting fees from Guidepoint Global and travel support from Forest Research Institute. Dr. Mago has also received research grants from Bristol Myers Squibb, Forest Research Institute, Shire, Otsuka, and Eli Lilly. Giovanno Forero, William M. Greenberg, Carl Gommoll, and Changzheng Chen are employees of Forest Research Institute, a subsidiary of Forest Laboratories, Inc.

We would like to acknowledge writing assistance and editorial support for the preparation of this manuscript was provided by Adam Ruth, PhD, and Carol Dyer, MS, of Prescott Medical Communications Group, Chicago, IL, USA, contractors of Forest Research Institute.

Open Access This article is distributed under the terms of the Creative Commons Attribution Noncommercial License which permits any noncommercial use, distribution, and reproduction in any medium, provided the original author(s) and the source are credited.

\section{References}

1. Mathers CD, Loncar D. Projections of global mortality and burden of disease from 2002 to 2030. PLoS Med. 2006;3(11):e442.

2. APA practice guideline for the treatment of patients with major depressive disorder, 3rd ed. Arlington: American Psychiatric Association; 2010. http://psychiatryonline.org/content.aspx? bookid=28\&sectionid=1667485. Accessed 19 Aug 2013 .

3. Kelly K, Posternak M, Alpert JE. Toward achieving optimal response: understanding and managing antidepressant side effects. Dialogues Clin Neurosci. 2008;10(4):409-18.

4. Auclair AL, Martel JC, Assié MB, et al. Levomilnacipran (F2695), a norepinephrine-preferring SNRI: profile in vitro and in models of depression and anxiety. Neuropharmacology. 2013; 70:338-47.

5. Stahl SM, Grady MM, Moret C, et al. SNRIs: their pharmacology, clinical efficacy, and tolerability in comparison with other classes of antidepressants. CNS Spectr. 2005;10(9):732-47.

6. Blier P, Saint-Andre E, Hebert C, et al. Effects of different doses of venlafaxine on serotonin and norepinephrine reuptake in healthy volunteers. Int $\mathbf{J}$ Neuropsychopharmacol. 2007;10(1): 41-50.

7. Deecher DC, Beyer CE, Johnston G, et al. Desvenlafaxine succinate: a new serotonin and norepinephrine reuptake inhibitor. J Pharmacol Exp Ther. 2006;318(2):657-65.

8. Forest Pharmaceuticals Inc. Fetzima ${ }^{\circledR}$ (levomilnacipran) extended-release capsules: U.S. Prescribing Information. St. Louis: Forest Pharmaceuticals, Inc.; 2013.

9. Chen L, Boinpally R, Gad N, et al. Drug-drug interactions of levomilnacipran sustained release capsule with ketoconazole, 
carbamazepine, or alprazolam in healthy subjects [poster]. 166th annual American Psychiatric Association Meeting; 2013 May 18-22; San Francisco.

10. Asnis G, Bose A, Gommoll C, et al. The efficacy and safety of levomilnacipran SR $40 \mathrm{mg}, 80 \mathrm{mg}$, or $120 \mathrm{mg}$ in major depressive disorder: a phase III, randomized, double-blind, placebocontrolled study. J Clin Psychiatry. 2013;74(3):242-8.

11. Bakish D, Gommoll C, Chen C, et al. Levomilnacipran ER $40 \mathrm{mg}$ and $80 \mathrm{mg}$ in major depressive disorder: a phase III, randomized, double-blind, fixed-dose, placebo-controlled study Int Clin Psychopharmacol. 2013 (in press).

12. Montgomery S, Mansuy L, Ruth A, et al. The efficacy and safety of levomilnacipran SR in major depressive disorder: a randomized, double-blind, placebo-controlled, proof-of-concept study. J Clin Psychiatry. 2013;74(4):363-9.

13. Sambunaris A, Bose A, Gommoll C, et al. W14. A phase III, double-blind, placebo-controlled, flexible-dose study of levomilnacipran SR in patients with major depressive disorder [abstract]. Neuropsychopharmacol. 2012;38(Suppl 1):S322-3.

14. Gommoll C, Bose A, Li H, et al. A randomized double-blind, placebo-controlled, flexible-dose study of levomilnacipran in patients with major depressive disorder [poster]. 24th Annual US Psychiatric and Mental Health Congress; 2011 Nov 7-10; Las Vegas.
15. Shiovitz T, Bose A, Greenberg WM, et al. The efficacy and safety of levomilnacipran SR in the prevention of relapse in major depressive disorder: results from a phase III clinical trial [poster]. 25th Annual US Psychiatric and Mental Health Congress; 2012 Nov 8-11; San Diego.

16. American Psychiatric Association (APA). Diagnostic and statistical manual of mental disorders. 4th ed. Text Rev. Washington, DC: American Psychiatric Association; 2000.

17. Montgomery SA, Åsberg M. A new depression scale designed to be sensitive to change. Br J Psychiatry. 1979;134:382-9.

18. Posner K, Brown GK, Stanley B, et al. The Columbia-Suicide Severity Rating Scale: initial validity and internal consistency findings from three multisite studies with adolescents and adults. Am J Psychiatry. 2011;168(12):1266-77.

19. Clinical Global Impressions. In: Guy W. ECDEU assessment manual for psychopharmacology. Rockville: National Institute of Mental Health; 1976, p. 218-222. DHEW publication no. 76-338.

20. Watkins PB, Seligman PJ, Pears JS, Avigan MI, Senior JR. Using controlled clinical trials to learn more about acute drug-induced liver injury. Hepatology. 2008;48(5):680-1689.

21. Cipriani A, Furukawa TA, Salanti G, et al. Comparative efficacy and acceptability of 12 new-generation antidepressants: a multiple-treatments meta-analysis. Lancet. 2009;373(9665):746-58. 